\title{
Conference Paper \\ Local Angiotensin Pathways in Human Carotid Atheroma: Towards a Systems Biology Approach
}

\author{
Giampiero Bricca, ${ }^{1,2}$ Liliana Legedz, ${ }^{1}$ Ali Nehme, ${ }^{1}$ Hanène Ayari, ${ }^{1}$ Christian Paultre, ${ }^{1}$ \\ Wassim Hodroj, ${ }^{1}$ Jacques-Yuan Li, ${ }^{1}$ Jacques Randon, ${ }^{1}$ Olivier Lohez, ${ }^{1}$ Nedra Dhaouadi, \\ Marie Paule Gustin, ${ }^{1}$ and Catherine Cerutti ${ }^{1}$

\begin{abstract}
${ }^{1}$ EA4173-Functional Genomics in Hypertension, Université de Lyon, Hôpital Nord-Ouest, Villefranche, Lyon, France
\end{abstract} \\ ${ }^{2}$ Service de Pharmacologie, INSERM U1088, Centre de Biologie Humaine, CHU Amiens, avenue Laennec, Salouel, \\ 80000 Amiens, France \\ Correspondence should be addressed to Giampiero Bricca; giampiero.bricca@inserm.fr
}

Received 19 June 2014; Revised 21 December 2014; Accepted 30 December 2014

Academic Editor: Antonio Lepedda

This Conference Paper is based on a presentation given by Giampiero Bricca at "LIAC Meeting on Vascular Research 2013" held from 18 September 2013 to 21 September 2013 in Alghero, Italy.

Copyright (C) 2015 Giampiero Bricca et al. This is an open access article distributed under the Creative Commons Attribution License, which permits unrestricted use, distribution, and reproduction in any medium, provided the original work is properly cited.

We will summarize the data we have obtained in human carotid artery concerning the organization of an extended local renin angiotensin aldosterone system and its variations at different stages of atheroma. In a system view, we propose a model where concomitant increase in angiotensin and glucocorticoid signaling is induced and amplified in VSMC while vascular smooth muscle cells transdifferentiate toward a lipid storing phenotype.

\section{Introduction}

Systems are complex objects linking several single components which, associated in a certain way with multiple relationships perform a specific task. Systems are submitted to many external inputs and their output expresses their function. Biological systems have been conceived in the frame of homeostasis and of the general systems theory using cybernetics for their investigation [1].

In the cardiovascular system, such a mechanistic, "engineer" representation is provided by the model of Guyton $[2,3]$ and its successful predictions [4]. However, if short-term evolutions may realistically be described and predicted, their power decreases quickly with the variety of initial conditions and over time probably because they are incomplete both in terms of knowledge implemented in the models and of current knowledge. Notably, the constant interplay between functional (responsiveness to different stimuli) and structural (cellular populations and extracellular matrix composition) properties that does take place in vivo lacks both knowledge and conceptual frame for modeling. Blood pressure or blood flow, glycemia, temperature, and most functionally relevant parameters measured or assessed by the physicians appear only at a high level of biological integration. These parameters result from numerous molecular events which take place at the cellular level in a large variety of tissues and are indeed coordinated through regulatory networks.

The knowledge of the complete human genome has shown surprisingly a rather "limited" amount of protein coding genes, less than 30000 . But, with several different transcripts per gene and several proteins per transcript, each subject to different posttranslational modifications, the complexity is largely enhanced. However, although high, these numbers are now reachable. We can count and classify genes according to various points of view. There are about 450 G-protein receptors coding genes, 561 coding for proteases, 161 for proteases inhibitors, and so on. Similarly, it is now possible to get a step further and explore quantitatively 


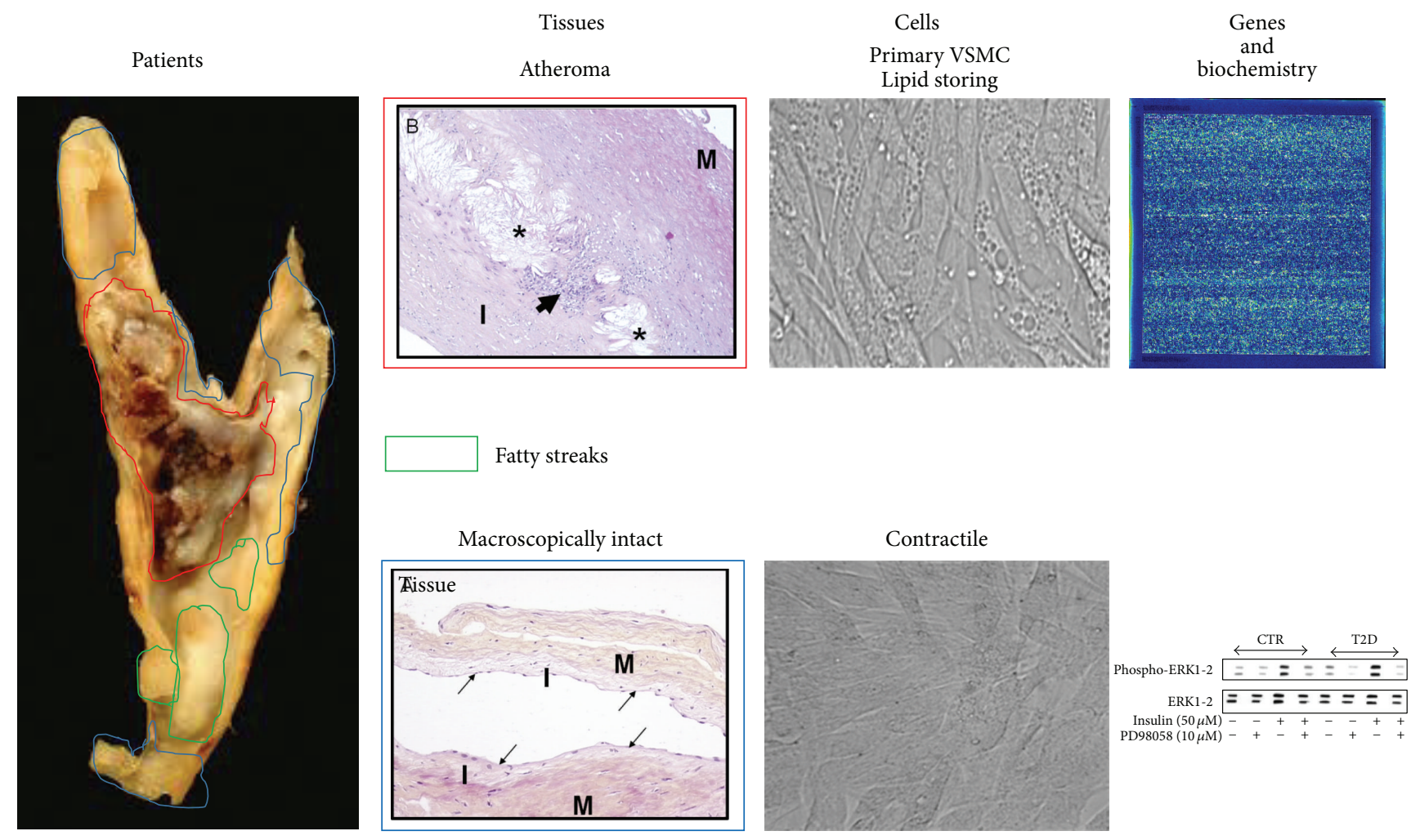

FIGURE 1: Human carotid atheroma as a study model of atheroma development. On the macroscopic view are drawn the dissections performed in a very remodeled carotid artery. Most often the three types of tissues, red: atheroma plaque, green: fatty streaks, and blue: macroscopically intact tissue, are more easy to delineate. Standard histological control confirms the grade of atherosclerotic remodeling higher than grade IV according to the classification of Stary for atheroma and lower than II for macroscopically intact tissue and tissues may be used for mRNA in situ hybridization and immunohistochemistry. From microscopically intact tissue, primary culture of vascular smooth muscle cells may be established and the responses are studied according to different phenotype in which cells can be conducted, "contractile" and "lipid storing." Interindividual variability and reproducibility of biochemical parameters may be assessed both in situ from the different segments dissected and from primary cells.

the amount of almost each transcribed RNA and all mRNA and this can be related to chromatin structure and DNA binding proteins (ENCODE).

This new knowledge allows a new paradigm where, instead of looking for a single component and its involvement in a specific step of a mechanism or pathway, it is possible to check among all of the possible known components and their interactions, those that do actually take place in a specific instance at a specific time point. Thus the questions of determinism and functional consequences are shifted from the possibility of one particular interaction to participate in the studied process to the identification of the set of components and interactions that are indeed present and active in the process in a specific situation.

The renin angiotensin aldosterone system (RAAS), including numerous enzymes, active or inactive peptides, and receptors, is one of these systems. Here, we will summarize according to this point of view some of the data we have obtained concerning the organization of the RAAS system in our study model of human carotid atheroma (Figure 1).

\section{Renin Angiotensin Aldosterone System}

The RAAS is an important endocrine system involved in blood pressure and blood volume regulation in normal and pathological situations. It is one of the major pharmacological targets in cardiovascular medicine with pharmacological classes as important as ACE inhibitors, angiotensin II type 1 and aldosterone receptors antagonists, and the recently introduced renin inhibitors. Its contribution to blood pressure regulation has even been summarized as a set of differential equations [5] and introduced into the model of Guyton. With respect to cardiovascular adverse remodeling and its associated morbidity, the RAAS "behaves" as a nonspecific amplifier of risk factors such as hypertension and insulin resistance and a local amplifier directly inducing deleterious cardiac and vascular remodeling [6,7]. Numerous reviews have been recently published emphasizing new aspects of the RAAS including prorenin/renin receptor, other active peptides, new enzymatic pathways, and new cellular mechanisms $[8,9]$.

In its cardiovascular endocrine system perspective, the tightly regulated kidney released plasma renin concentration 
is the rate-limiting step of Ang II generation starting from circulating angiotensinogen (AGT). AGT delivered in the plasma by the liver and possibly adipose tissue circulates at concentrations around or lower than the $\mathrm{Km}$ for renin and has an estimated plasma half-life of several hours decreased by renin $[10,11]$. AGT has no acute regulatory properties, but its basal expression may participate in the long-term regulation of RAAS activity [12, 13]. ACE is a non-rate-limiting enzyme abundantly expressed on almost all endothelial cells with a soluble form circulating in the plasma [14]. The RAAS is thus a peculiar endocrine system because the key regulated component is not the release of the active form of the hormone, but that of an enzyme. The high specificity of the response relies on the high selectivity and efficiency of the protease activity of renin toward AGT which is its only known substrate. But this opens the possibility that Ang II may be produced through proteolytic cleavage of AGT by other proteases. Indeed, neither the complete blockade of the plasma renin nor the complete inhibition of ACE and even their association could lead to complete disappearance of plasma Ang II, indicating that a "leak" exists somewhere and that other enzymatic pathways are accessible $[15,16]$. This "leak" represents the background over which renal renin dependent Ang II production produces its effects and is a constant component of the effects of pharmacological inhibition of angiotensin signaling.

\section{Extended RAAS: from Endocrine to Paracrine-Tissue Angiotensin Systems}

Numerous tissues, brain, kidney, heart, ovary, pancreas, and vascular wall have now been shown to express a functional paracrine angiotensin system. This usually refers to the ability of producing angiotensin II with renin-like activity and responding to angiotensin II independently of the kidney release of renin [17, 18]. From the canonical 2 enzymatic steps with renin and ACE in plasma, numerous enzymes have been shown to be able to participate in tissue angiotensin systems. Indeed, up to now, at least 18 peptidases have been shown to participate in angiotensin peptide generation and metabolism (Figure 2(a)) $[8,19]$. In these networks of proteases, "competition" between proteolytic pathways may occur as was recently shown for ACE and ACE 2 competing for Ang I in the kidney $[19,20]$ or between different substrates for the same enzymes such as ACE with Ang I and kinins. Indeed the kinins pathway is closely interlinked with angiotensin system both at the functional and biochemical levels. The kinins pathway resembles the angiotensin system in that it is a signaling peptides cascade generated from a single-gene precursor protein "kininogen" and a set of enzymes. Some enzymes such as tissue kallikrein (KLK1), neprilysin (MME), or ACE are shared with the angiotensinogen cascade. Numerous effects of ACE inhibition have been related to increasing kinins signaling [21] and receptors have been shown to interact in the cell membrane [22]. Tissue analysis of renin angiotensin systems has also revealed the existence of other active peptides, angiotensin 1-7 and Ang IV with specific receptors, respectively, Mas1 and AT4R (insulin regulated aminopeptidase IRAP, LNPEP, and receptors for enzymes such as renin $[18,23]$. These elements should also be taken into account when considering the tissue organization and function.

Because aldosterone, the mineralocorticoid of adrenal origin, the synthesis and secretion of which are highly dependent on Ang II stimulation through AT1R receptors, contributes to the overall cardiovascular effects of the RAAS activation, it is often considered part of the system. Aldosterone participates in these actions through its receptor MR to induce salt retention and direct cardiac and vascular actions. However, in a system view, including aldosterone in the system brings together the whole mineralo- and glucocorticoids pathways (Figure 2(b)). Indeed, cortisol, in the absence of the MR protecting enzyme $11 \beta$-hydroxysteroid dehydrogenase type 2 (HSD11B2), can stimulate MR [24] and cortisol may be produced locally through NADPH dependent activity of HSD11B1 from the inactive cortisone [25]. Importantly, in a paracrine system view, HSD11B1 has been shown to play a major role in lipid storing tissues to induce a metabolic syndrome with high blood pressure and high AGT in mice [26] and is present in various other tissues and notably the arterial wall [24]. Moreover, glucocorticoids have been shown in several instances to increase tissue RAAS gene transcription and, in patients, the endogenous or exogenous excess in glucocorticoids is associated with hypertension, vascular remodeling, and increased cardiovascular risk [27]. Several authors have hypothesized a close physiopathological link between local angiotensin and corticoid systems in the adipose tissues and the development of a metabolic syndrome [28].

A set of 39 candidate genes which may contribute to an extended tissue RAAS can be established (Figure 2, Table 1). The whole system can be classified according to substrates (2 genes), proteases (18 genes), steroid metabolizing enzymes (9 genes), and receptors (10 genes). It may be hypothesized that tissue-specific RAAS organizations will refer to specific subsets of these potential participants.

\section{Human Carotid Atheroma Extended RAAS}

In our atheroma studies we have investigated individually 22 out of the 39 genes of the extended tRAAS in our model of human carotid atheroma (Table 1).

4.1. Substrates. For angiotensin and kinin signaling, the substrates are derived from a single-gene product, respectively, angiotensinogen (AGT) and kininogen (KNG1); for cortisol and aldosterone, the substrate, cholesterol, is not directly gene-coded.

As in other vascular models, the mRNA for AGT can be detected in the medial vascular smooth muscle cells (VSMC) from human carotid arteries and significant amounts of AGT protein can be detected in the arterial wall $[29,30]$. The substrate is present and locally synthesized in human carotid atheroma at almost similar levels in advanced stages of atheroma and low grade remodeling in human carotid [31]. AGT expression and content of low grade remodeled carotid arteries was significantly higher in type 2 diabetic patients 

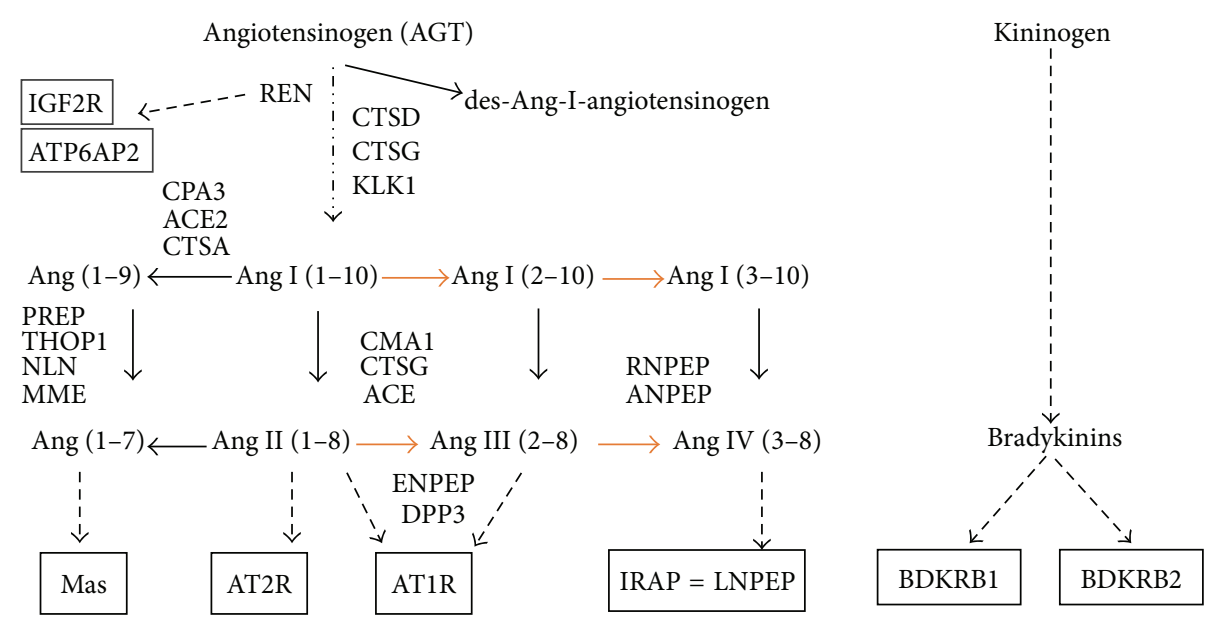

(a)

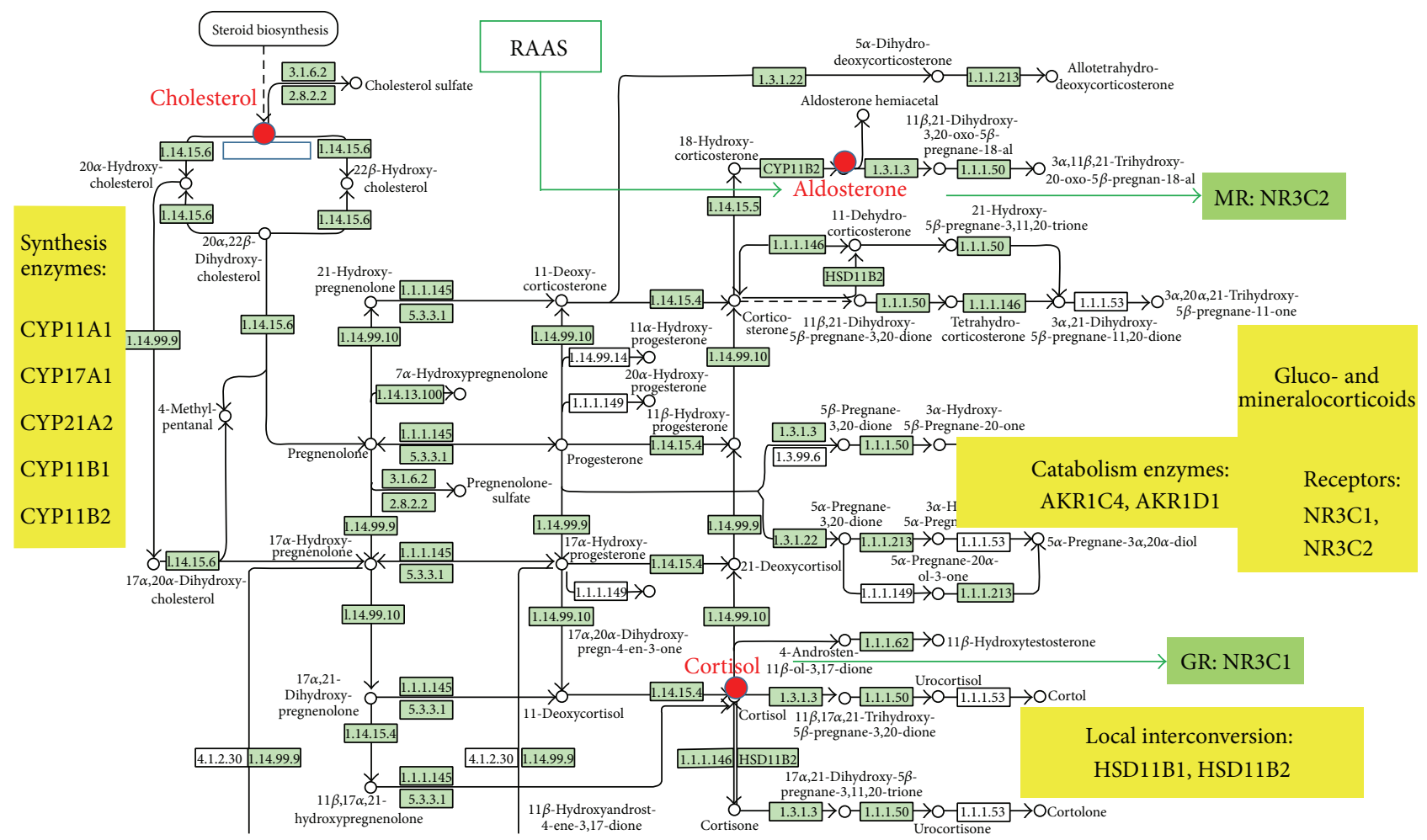

(b)

Figure 2: Extended RAAS. (a) The peptidases able to perform Ang II synthesis and the peptidases involved in the generation of the other potential active peptides Ang 1-7 and Ang IV have been added to the classical RAAS system, a schematic bradykinin pathway including kininogen, the bradykinins receptors B1 and B2. The gluco- and mineralocorticoids signaling comprises enzymes and receptors. (b) The cortico- and mineralocorticoid pathways components. Although the number of metabolites is high, the number of enzymes and receptors is rather limited. 39 potential contributors to any tissue extended RAAS taking into account kinin precursor and receptors and a complete gluco/mineralocorticoid pathways were considered. Adapted from KEGG database: http://genome.jp/kegg/pathway/map/map00140.html.

suggesting a link between systemic risk factors and local activation of AGT expression in the arterial wall. We showed that this overexpression in type 2 diabetic patients could result from the imbalance in favor of the MAP kinase pathway over the PI3 kinase pathway seen in advanced atheroma stages and associated with insulin responses and insulin resistance [32]. Kininogen mRNA was not detected in the human carotid artery samples while some immunohistochemical reactivity could be detected in the most luminal regions suggesting the possibility of a limited plasmatic supply [33].

4.2. Signal Generation. The active hormone(s) are produced directly or indirectly from the primary substrate AGT. In the RAAS system the signaling peptides are produced from 
TABLE 1: Extended RAAS genes investigated in human carotid atheroma investigated with RT-PCR (mRNA), western blotting, and/or ELISA (protein) and enzymatic activity (*); ND: not detected.

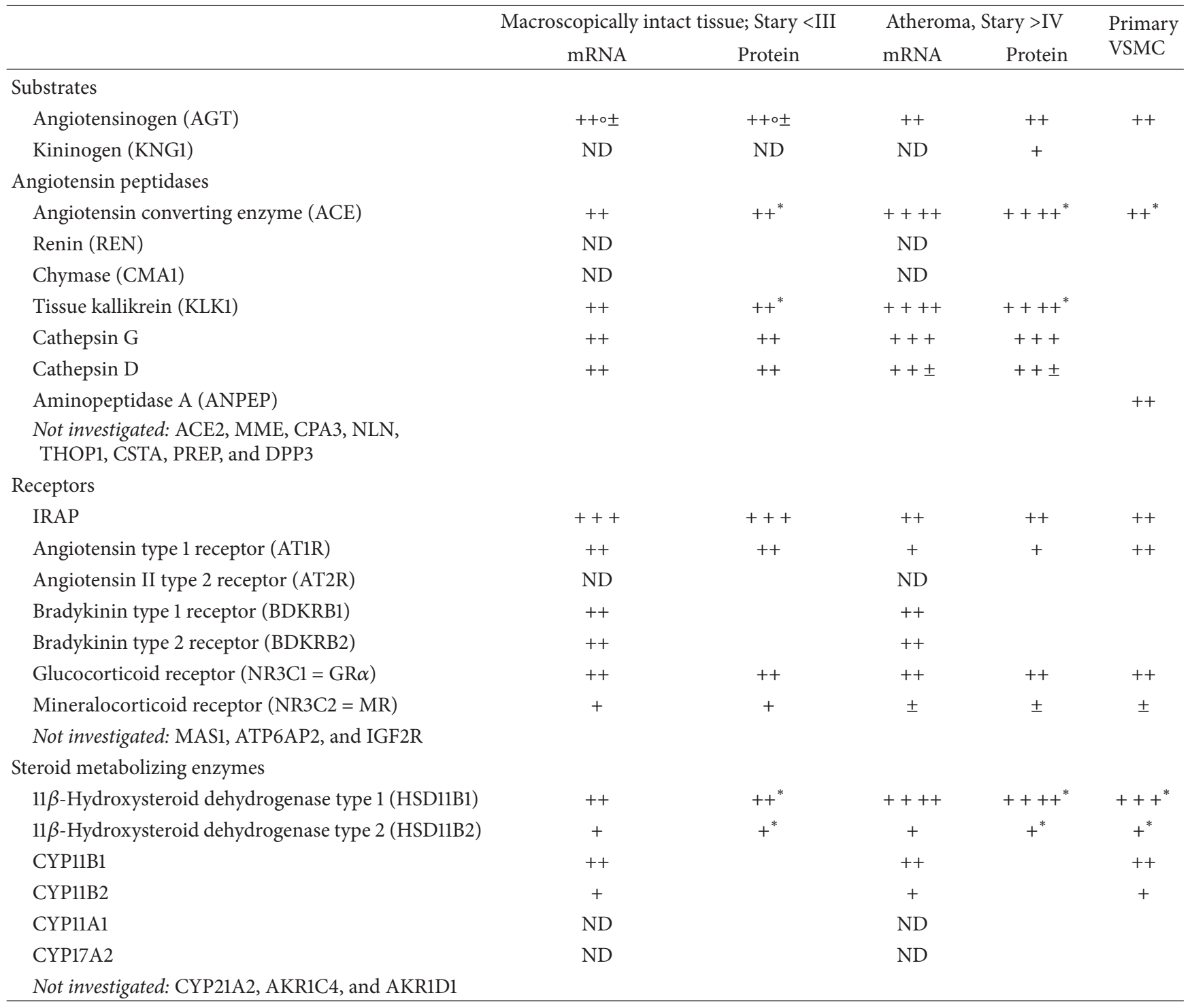

a major intermediate Ang 1 (1-10) and to date at least 18 peptidases, producing Ang 1 or cleaving Ang 1 into smaller fragments, are recognized (Figure 1 and Table 1). The recent unraveling that Ang 1-12 and Ang 1-14 may become intermediate substrate for signaling peptide generation will probably widen the spectrum of proteases to be considered. All enzymes, except renin, accept several substrates opening possibilities for interactions with other pathways such as the elastin pathway where the angiotensin 1-9 generating cathepsin $\mathrm{A}$ is part of the newly described elastin peptide receptor complex and where cathepsin $G$ and $M M E$ are potent elastases.

The capacity of blood vessels and vascular smooth muscle cells to produce angiotensin II from local AGT and Ang I independently of kidney released renin has been abundantly documented [34]. ACE is expressed by vascular endothelial cells and also by human VSMC from carotid endarterectomy samples. We have found that ACE mRNA and ACE activity increased severalfold in the advanced stages of atheroma plaque compared to low level remodeling confirming previous immunohistochemical arguments in favor of local Ang II overproduction capacity.

The presence of renin mRNA in the arterial wall, probably because of disparity of different vascular beds and whether adventitia is considered or not, is controversial. Indeed, we could not detect renin mRNA neither in low nor in high grade atheroma remodeling from human carotid endarterectomy. Similarly, chymase (CMA1) could not be measured reliably, suggesting low, if any, expression of these enzymes in the carotid vascular wall [31]. We have investigated several alternative angiotensin production pathways. Tissue kallikrein (KLK1) can produce both Ang I and Ang II directly from AGT and generate kinins from kininogen. Cathepsin D (CTSD) is able to generate Ang I from AGT and cathepsin G (CTSG) 
can form Ang II from Ang I. ACE, KLK1, and CTSG are much more expressed and active in the advanced stages of atheroma, indicating that a complete set of angiotensin II forming enzymes may operate directly from AGT in the atherogenic process.

The degradation of angiotensin II is either through carboxypeptidases (ACE2, MME, and NLN) leading to Ang 1-7 and smaller peptides or through aminopeptidases (ANPEP, ENPEP, and DPP3) leading to Ang IV (3-8) and smaller fragments. These enzymes have poor selectivity and accept a wide range of substrate such as kinins, endorphins, oxytocin, and so on. ACE2 and the Ang 1-7 pathway has attracted much attention [18]. ACE2 gene knockout in mice appeared to favor atheroma development [35] and overexpression in rabbits stabilizes the plaques [36]. In human ACE2 has been shown to be increased in atheroma from diabetic patients [37]. Aminopeptidase $\mathrm{N}$ and aminopeptidase A (ANPEP, ENPEP) producing sequentially angiotensin III (3-8) and angiotensin IV (4-8) are also able to degrade kinins but have yet been poorly investigated in the arterial wall and atheroma [38]. Dipeptidyl peptidase 3 (DPP3) may also directly produce angiotensin IV and degrade angiotensin III into smaller fragments. IRAP insulin regulated aminopeptidase (LNPEP) is also able to produce Ang IV from Ang III and may be considered from both sides of receptors and enzyme. From preliminary analysis of publicly available transcriptomic data on atheroma presented at the meeting, while very low levels of AC2, ANPEP, and ENPEP were detected, DPP3, MME, and NLN were in the median range of mRNA abundance.

4.2.1. Angiotensin 1-7. The pathway toward angiotensin 1-7 formation may start from Ang I or Ang II. The main enzyme is ACE2 which not only produces Ang 1-9, competing with ACE for Ang I, but also degrades angiotensin II in Ang 17 (Figure 3). In the Ang 1-9-Ang 1-7 pathway, several other peptidases, elastase- 2 = MME, THOP1, NLN, and PREP, have been shown to display consistent $\mathrm{Km}$ for angiotensin I or angiotensin 1-9 and could also participate in a tissue angiotensin system. From publicly available transcriptomic data, most of these enzymes may be present in the arterial wall but ACE2 seemed in the very low range level of expression. Their variations according to stages of atheroma could not be addressed yet.

4.3. Gluco- and Mineralocorticoid Metabolizing Enzymes. The local content of cortisol and aldosterone depends not only on the supply through circulation and adrenal release but also on local metabolism involving complete synthesis and/or through local cortisol-cortisone interconversion by hydroxysteroid dehydrogenases B type 2 (HSD11B2) and type 1 (HSD11B1). The former transforms the active cortisol in inactive cortisone and protects MR from cortisol actions while the latter (HSD11B1) catalyzes the reverse reaction and amplifies locally the cortisol concentration and effects.

Cyp11A1 and CYP 17 mRNA coding for enzymes involved in the initial steps from cholesterol could not be detected in human carotid atheroma. mRNA coding for the CYP11B1 and CYP11B2, involved in de novo biosynthesis of cortisol and aldosterone from progesterone, HSD11B1, and HSD11B2, could be reliably and regularly measured as well. The absolute number of copies measurements showed that the mineralocorticoid pathway components CYP11B2 and HSD11B2 mRNA were at much lower levels than the cortisol synthesizing enzymes HSD11B1 and CYP11B1 mRNA. HSD11B1 was found at much higher levels and the cortisone-cortisol conversion was sharply increased in advanced stages of atheroma showing in the atherosclerotic process the importance of local cortisol production through cortisone reduction [39]. In human carotid primary VSMC, these steroid metabolizing enzymes are expressed and subject to differential regulation as shown with the shift from a contractile to a lipid storing phenotype associated with much higher levels of CYP11B1, CYP11B2, and HSD11B1 in the lipid storing VSMC. Overall a major increase in cortisol and aldosterone signaling appears during atherogenesis [39].

4.4. Signal Reception: Receptors. The signaling molecules resulting from extended RAAS may act through different receptors. Five are G-protein-coupled receptors: 2 for Ang II and/or Ang III (AGTR1 and AGTR2), 1 for Ang 1-7 (MAS), and 2 for kinins B1 and B2 (BDKRB1, BDKRB2). The mineralo- and glucocorticoid receptors $(M R=N R 3 C 2$ and $\mathrm{GR} \alpha=\mathrm{NR} 3 \mathrm{C} 1$ ) belong to the family of transcription factors receptors. The proposed renin receptor IGF2R belongs to the receptor kinase family while the other renin-prorenin receptor (ATP6AP2) is atypical as is also the putative Ang IV receptors IRAP (LNPEP).

In the human carotid artery, we could reliably detect and quantify levels of expression of AGTR1, GR, MR, BDKRB1, BDKRB2, and IRAP, but we could not detect AT2R mRNA. The preliminary analysis of atheroma transcriptomic public data suggests that MAS mRNA as AT2R mRNA might be close to background while IGF2R and ATP6AP2 were present and among the most abundant mRNAs. AT1R, MR, and IRAP mRNAs and the corresponding proteins were found at lower levels in atheroma while the $\mathrm{B} 2$ bradykinin receptor mRNA was moderately increased.

\section{Extended tRAAS as a Functional System in Atheroma}

In order to consider the functional organization, we have investigated the reactivity of some of these components in primary vascular smooth muscle cells obtained from the macroscopically intact part of carotid endarterectomy samples. AGT expression by human VSMC was influenced by diabetes but was neither modified by high glucose nor modified by the antidiabetic metformin, while it was strongly stimulated by insulin and decreased by glibenclamide [32]. Ang II decreased while Ang IV and cortisol strongly increased by more than 3-fold AGT expression by primary human carotid VSMC (Hodroj unpublished results) and the transition from "contractile" to lipid storing phenotype was associated with increased AGT expression [40].

The existence of correlations between mRNA amounts of enzymes and the existence of strong positive correlations between GR and MR further suggested the existence of a 


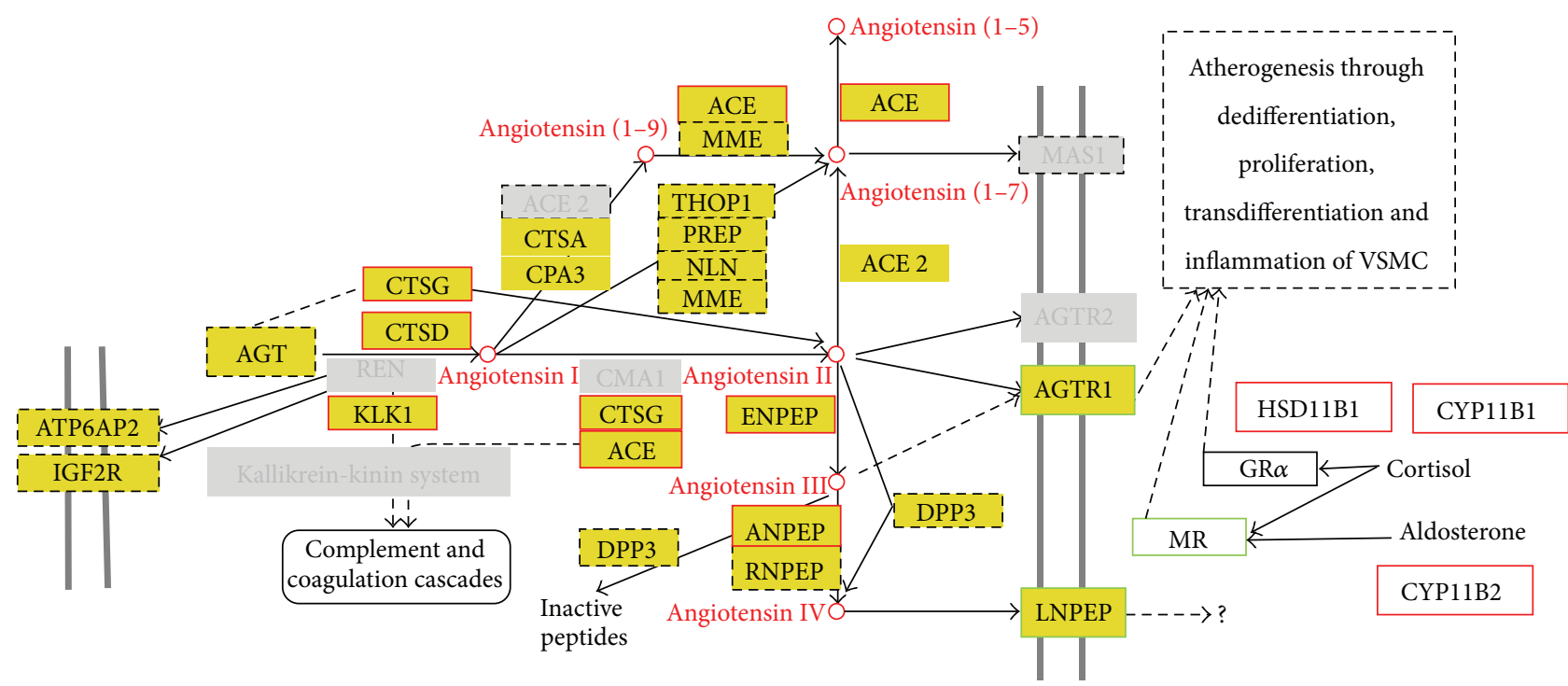

FIGURE 3: Extended RAAS in the human carotid atheroma. Boxes delineate investigated gene products, in black (similar levels), red (higher levels), and green (lower levels) in advanced stages of atheroma compared to low grade remodeling. Shaded parts are those which were at too low level for reliable quantification. Interrupted frames are those for which publicly available human carotid atheroma transcriptomic data indicated the presence of very low levels. Adapted from KEGG database: http://genome.jp/kegg/pathway/map/map04614.html.

coordinated regulation during atherogenesis with synergistic variations toward the facilitation of Ang II and Ang IV formation and responses in the atherosclerotic arterial wall. In human cultured VSMC from carotid artery, aminopeptidase A and IRAP expression in human VSMC were both increased by low nanomolar concentrations of Ang II and both decreased by Ang IV (Hodroj unpublished results). Investigations into transcriptomic public databases of human carotid atheroma confirm the presence of high or very high levels of ANPEP, ENPEP, IRAP, and DPP3 mRNA in human carotid atheroma.

In cultured primary human carotid VSMC, ACE mRNA was insensitive while CTSD mRNA was increased by more than 3-fold by Ang IV and decreased by Ang II (Hodroj unpublished). ATIR mRNA level was stimulated by insulin and cortisol and decreased by glibenclamide but unaffected by high glucose or metformin. Angiotensin II and angiotensin IV acted differentially, the first decreasing and the second increasing ATIR mRNA (Hodroj unpublished). GR and MR were present in primary human VSMC and were differentially regulated by cortisol and aldosterone. Cortisol increased the expression of GR while decreasing MR through illicit activation of MR. Fludrocortisone as selective agonist of MR decreased MR expression only. The lipid storing transition of VSMC from a contractile to a lipid storing phenotype was accompanied by a sharp decrease in MR expression [39]. Despite being at low levels, MR was active and could display differential responses according to VSMC phenotype as seen with collagen A1 which is induced by fludrocortisone only in lipid storing phenotypes. Variations in receptor number may be interpreted according to signaling systems where the receptor response is maintained at different levels of stimuli through opposite variations of receptor number and signal strengh. In such a frame, the sharply lower levels of AT1R and MR may result from a higher stimulation, while the slight increase in bradykinin $\mathrm{B} 2$ receptor would be the witness for lower stimulation. However, another not exclusive explanation could lie in the transdifferentiation of VSMC from a lipid to a storing lipid phenotype, as seen with the lower MR in adipocyte transdifferentiated VSMC.

Based on these results, we can now try to draw a picture of the extended RAAS functioning in atheroma (Figure 3). The presence of a local expression of AGT indicates that the system may be fuelled by local supply, which seems not to be the case for the kinins system. Local AGT could be influenced by risk factors such as type 2 diabetes, insulin resistance, and high cortisol and may thus behave as a local nonspecific amplifier of risk factors. Among the 6 possible angiotensin II forming enzymes, two, renin and chymase, are not found, while the others ACE, CatD, CatG, and tissue kallikrein are present and active and 3 of them are overexpressed and hyperactive in advanced stages of carotid atheroma. A high local concentration of cortisol seems to be possible mostly thanks to the high expression of HSD11B1. Aldosterone synthesis seems possible from pregnenolone, but, due to the low level of HSD11B2, illicit activation of MR by cortisol is also possible. The double activation of MR by aldosterone and cortisol may explain the strong downregulation observed in advanced stages of carotid atheroma and during the lipid storing transition of VSMC.

The bradykinin synthesis should be low due to a local low supply of substrate restricted to diffusion from the vascular lumen and the presence of high amounts of the degrading enzymes ACE and aminopeptidase. In terms of responsiveness we could show the absence of downstream bradykinin receptor transduction differential activation according to 
the grade of atherosclerotic remodeling arguing against an activation of the bradykinin B1 or B2 transduction pathway through GMPc and G-kinase in atheroma.

In terms of tissue responsiveness, it is noteworthy that the AT2R could not be detected and that IRAP as a potential Ang IV receptor was abundantly found and appeared to be downregulated, as was the case for ATIR and MR in atheroma. Moreover, the existence of human VSMC differential responses to Ang II and Ang IV in VSMC strongly argues in favor of an active Ang IV specific pathway in atheroma possibly involving IRAP.

Cortisol increases HSD11B1 through GR activation and decreases HSD11B2 through both GR and MR activation. It also favors the expression of its own receptor GR and through the same receptor triggers the expression of vascular remodeling factors such as collagen $1 \mathrm{Al}, \mathrm{MCP} 1$, and FAT4.

From a physiopathological point of view this suggests that a kind of vicious circle between angiotensin and steroid signaling amplifying each other may be established at the initial stages of vascular remodeling favoring both dedifferentiation and atheroma formation. Atheroma formation through the transdifferentiation of VSMC may further accelerates this vicious circle.

\section{Conclusion}

Thus in carotid atheroma development a specific combination of peptidergic enzymes, cortisol metabolizing enzymes, and receptors can be identified. Whether this combination is a specific atheroma feature has to be determined in full detail. Thanks to the availability of public transcriptomic data (shown at the meeting), preliminary results obtained in different normal tissues from human confirm the hypothesis that a description and classification of tissue specific organization of tRAAS according to mRNA coexpression of its components may be achieved.

The multiple interactions between the components of the extended tRAAS in atherosclerotic lesions reinforce the necessity of conducting global approaches of systems biology to better understand the function, dysfunction, and pharmacology of this complex system. Thanks to the development of mass spectrometry of proteins, we can now consider the possibility of precisely measuring simultaneously in the same biological sample tens if not hundreds of selected proteins or peptides and of easily performing these measurements in numerous patients and tissues. It is thus feasible and necessary to accurately quantify the amounts of protein, mRNA, and flows of signaling peptides and steroid production in the low level and advanced atheroma as well as in vascular smooth muscle cells according to their actual phenotype. The identification of the mechanisms underlying a specific atheroma RAAS would then pave the way for atheroma specific therapeutic strategies.

\section{Conflict of Interests}

The authors declare that there is no conflict of interests regarding the publication of this paper.

\section{Acknowledgments}

This work has been supported over time by the CHU Grenoble, INSERM, Université Claude Bernard Lyon 1, Région Rhône-Alpes, SFHTA, Fondation de France, Fondation pour la Recherche Médicale, Fondation de l'Athérothrombose, and Hôpital Nord-Ouest, Villefranche.

\section{References}

[1] L. von Bertalanffy, General System Theory: Foundations, Development, Applications, George Braziller, New York, NY, USA, 1968.

[2] A. C. Guyton, T. G. Coleman, and H. J. Granger, "Circulation: overall regulation," Annual Review of Physiology, vol. 34, pp. 1344, 1972.

[3] R. Moss, T. Grosse, I. Marchant, N. Lassau, F. Gueyffier, and S. R. Thomas, "Virtual patients and sensitivity analysis of the Guyton model of blood pressure regulation: towards individualized models of whole-body physiology," PLoS Computational Biology, vol. 8, no. 6, Article ID e1002571, 2012.

[4] J. P. Montani and B. N. van Vliet, "Understanding the contribution of Guyton's large circulatory model to long-term control of arterial pressure," Experimental Physiology, vol. 94, no. 4, pp. 382-388, 2009.

[5] K. Claassen, S. Willmann, T. Eissing, T. Preusser, and M. Block, "A detailed physiologically based model to simulate the pharmacokinetics and hormonal pharmacodynamics of enalapril on the circulating endocrine renin-angiotensin-aldosterone system," Frontiers in Physiology, vol. 4, article 4, 2013.

[6] D. T. Lackland, "Hypertension: joint national committee on detection, evaluation, and treatment of high blood pressure guidelines," Current Opinion in Neurology, vol. 26, no. 1, pp. 812, 2013.

[7] R. S. McKelvie, G. W. Moe, J. A. Ezekowitz et al., "The 2012 Canadian Cardiovascular Society heart failure management guidelines update: focus on acute and chronic heart failure," Canadian Journal of Cardiology, vol. 29, no. 2, pp. 168-181, 2013.

[8] C. Becari, E. B. Oliveira, and M. C. O. Salgado, "Alternative pathways for angiotensin II generation in the cardiovascular system," Brazilian Journal of Medical and Biological Research, vol. 44, no. 9, pp. 914-919, 2011.

[9] I. Herichova and K. Szantoova, "Renin-angiotensin system: upgrade of recent knowledge and perspectives," Endocrine Regulations, vol. 47, no. 1, pp. 39-52, 2013.

[10] U. Hilgenfeldt, "Half-life of rat angiotensinogen: influence of nephrectomy and lipopolysaccharide stimulation," Molecular and Cellular Endocrinology, vol. 56, no. 1-2, pp. 91-98, 1988.

[11] J. Bohlender, J. Ménard, D. Ganten, and F. C. Luft, "Angiotensinogen concentrations and renin clearance: implications for blood pressure regulation," Hypertension, vol. 35, no. 3, pp. 780-786, 2000.

[12] D. B. Gordon, "The role of renin substrate in hypertension," Hypertension, vol. 5, no. 3, pp. 353-362, 1983.

[13] X. Jeunemaitre, "Genetics of the human renin angiotensin system," Journal of Molecular Medicine, vol. 86, no. 6, pp. 637641, 2008.

[14] R. A. Gonzalez-Villalobos, X. Z. Shen, E. A. Bernstein et al., "Rediscovering ACE: novel insights into the many roles of the angiotensin-converting enzyme," Journal of Molecular Medicine, vol. 91, no. 10, pp. 1143-1154, 2013. 
[15] J. D. Swales and H. Thurston, "Generation of angiotensin II at peripheral vascular level: studies using angiotensin II antisera," Clinical Science, vol. 45, no. 5, pp. 691-700, 1973.

[16] H. Thurston and J. D. Swales, "Action of angiotensin antagonists and antiserum upon the pressor response to renin: further evidence for the local generation of angiotensin II," Clinical Science and Molecular Medicine, vol. 46, no. 2, pp. 273-276, 1974.

[17] M. Paul, A. P. Mehr, and R. Kreutz, "Physiology of local reninangiotensin systems," Physiological Reviews, vol. 86, no. 3, pp. 747-803, 2006.

[18] M. Bader and D. Ganten, "Update on tissue renin-angiotensin systems," Journal of Molecular Medicine, vol. 86, no. 6, pp. 615621, 2008.

[19] K. Kramkowski, A. Mogielnicki, and W. Buczko, "The physiological significance of the alternative pathways of angiotensin II production," Journal of Physiology and Pharmacology, vol. 57, no. 4, pp. 529-539, 2006.

[20] M. C. Prieto, R. A. González-Villalobos, F. T. Botros et al., "Reciprocal changes in renal ACE/ANG II and ACE2/ANG 1-7 are associated with enhanced collecting duct renin in Goldblatt hypertensive rats," The American Journal of Physiology-Renal Physiology, vol. 300, no. 3, pp. F749-F755, 2011.

[21] E. Messadi-Laribi, V. Griol-Charhbili, E. Gaies et al., "Cardioprotection and kallikrein-kinin system in acute myocardial ischaemia in mice," Clinical and Experimental Pharmacology and Physiology, vol. 35, no. 4, pp. 489-493, 2008.

[22] P. C. Wilson, M.-H. Lee, K. M. Appleton et al., "The arrestinselective angiotensin AT1 receptor agonist [Sar1,Ile4,Ile8]-AngII negatively regulates bradykinin B2 receptor signaling via AT1B2 receptor heterodimers," The Journal of Biological Chemistry, vol. 288, no. 26, pp. 18872-18884, 2013.

[23] M. Ohishi, K. Yamamoto, and H. Rakugi, "Angiotensin (17) and other angiotensin peptides," Current Pharmaceutical Design, vol. 19, no. 17, pp. 3060-3064, 2013.

[24] G. A. Molnar, C. Lindschau, G. Dubrovska et al., "Glucocorticoid-related signaling effects in vascular smooth muscle cells," Hypertension, vol. 51, no. 5, pp. 1372-1378, 2008.

[25] J. R. Seckl and B. R. Walker, "Minireview: 11 $\beta$-hydroxysteroid dehydrogenase type 1-a tissue-specific amplifier of glucocorticoid action," Endocrinology, vol. 142, no. 4, pp. 1371-1376, 2001.

[26] H. Masuzaki, H. Yamamoto, C. J. Kenyon et al., "Transgenic amplification of glucocorticoid action in adipose tissue causes high blood pressure in mice," The Journal of Clinical Investigation, vol. 112, no. 1, pp. 83-90, 2003.

[27] L. Tauchmanovà, R. Rossi, B. Biondi et al., "Patients with subclinical Cushing's syndrome due to adrenal adenoma have increased cardiovascular risk," Journal of Clinical Endocrinology and Metabolism, vol. 87, no. 11, pp. 4872-4878, 2002.

[28] G. P. Vinson, "Angiotensin II, corticosteroids, type II diabetes and the metabolic syndrome," Medical Hypotheses, vol. 68, no. 6, pp. 1200-1207, 2007.

[29] S. Desjardins-Giasson, J. Gutkowska, R. Garcia, and J. Genest, "Renin substrate in rat mesenteric artery," Canadian Journal of Physiology and Pharmacology, vol. 59, no. 6, pp. 528-532, 1981.

[30] A. J. Naftilan, W. M. Zuo, J. Inglefinger, T. J. Ryan Jr., R. E. Pratt, and V. J. Dzau, "Localization and differential regulation of angiotensinogen mRNA expression in the vessel wall," The Journal of Clinical Investigation, vol. 87, no. 4, pp. 1300-1311, 1991.

[31] L. Legedz, J. Randon, C. Sessa et al., "Cathepsin G is associated with atheroma formation in human carotid artery," Journal of Hypertension, vol. 22, no. 1, pp. 157-166, 2004.
[32] W. Hodroj, L. Legedz, N. Foudi et al., "Increased insulinstimulated expression of arterial angiotensinogen and angiotensin type 1 receptor in patients with type 2 diabetes mellitus and atheroma," Arteriosclerosis, Thrombosis, and Vascular Biology, vol. 27, no. 3, pp. 525-531, 2007.

[33] L. Legedz, J. Randon, C. Sessa et al., "Induction of tissue kallikrein in human carotid atheroma does not lead to kallikrein-kinins pathway activation," Journal of Hypertension, vol. 23, no. 2, pp. 359-366, 2005.

[34] D. N. Müller, J. Bohlender, K. F. Hilgers et al., "Vascular angiotensin-converting enzyme expression regulates local angiotensin II," Hypertension, vol. 29, no. 1, pp. 98-104, 1997.

[35] M. Sahara, M. Ikutomi, T. Morita et al., "Deletion of angiotensin-converting enzyme 2 promotes the development of atherosclerosis and arterial neointima formation," Cardiovascular Research, vol. 101, no. 2, pp. 236-246, 2014.

[36] B. Dong, C. Zhang, J. B. Feng et al., "Overexpression of ACE2 enhances plaque stability in a rabbit model of atherosclerosis," Arteriosclerosis, Thrombosis, and Vascular Biology, vol. 28, no. 7, pp. 1270-1276, 2008.

[37] K.-R. Purushothaman, P. Krishnan, M. Purushothaman et al., "Expression of angiotensin-converting enzyme 2 and its end product angiotensin 1-7 is increased in diabetic atheroma: implications for inflammation and neovascularization," Cardiovascular Pathology, vol. 22, no. 1, pp. 42-48, 2013.

[38] J.-P. Fortin, L. Gera, J. Bouthillier, J. M. Stewart, A. Adam, and F. Marceau, "Endogenous aminopeptidase $\mathrm{N}$ decreases the potency of peptide agonists and antagonists of the kinin B1 receptors in the rabbit aorta," Journal of Pharmacology and Experimental Therapeutics, vol. 314, no. 3, pp. 1169-1176, 2005.

[39] H. Ayari, L. Legedz, P. Lantelme et al., "Auto-amplification of cortisol actions in human carotid atheroma is linked to arterial remodeling and stroke," Fundamental and Clinical Pharmacology, vol. 28, no. 1, pp. 53-64, 2014.

[40] H. Ayari, L. Legedz, C. Cerutti et al., "Mutual amplification of corticosteroids and angiotensin systems in human vascular smooth muscle cells and carotid atheroma," Journal of Molecular Medicine, vol. 92, no. 11, pp. 1201-1208, 2014. 


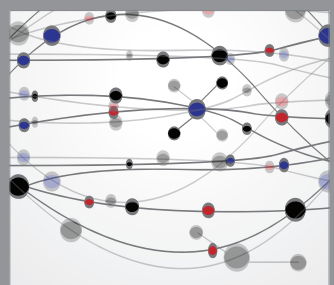

The Scientific World Journal
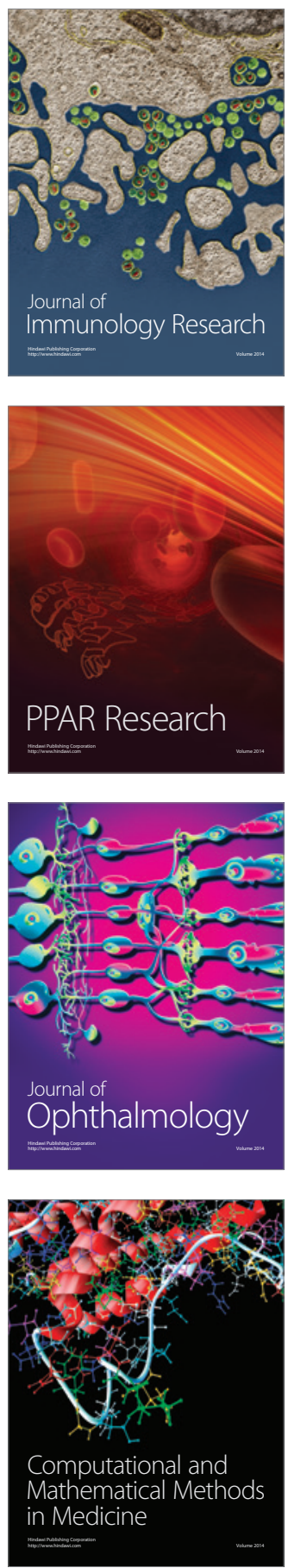

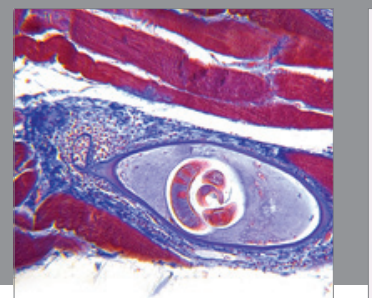

Gastroenterology

Research and Practice
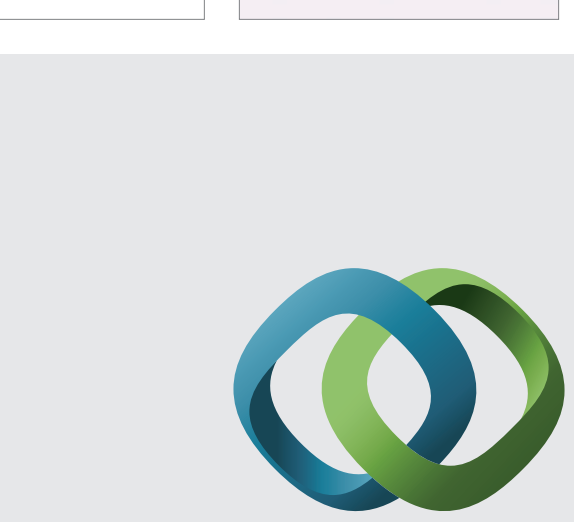

\section{Hindawi}

Submit your manuscripts at

http://www.hindawi.com
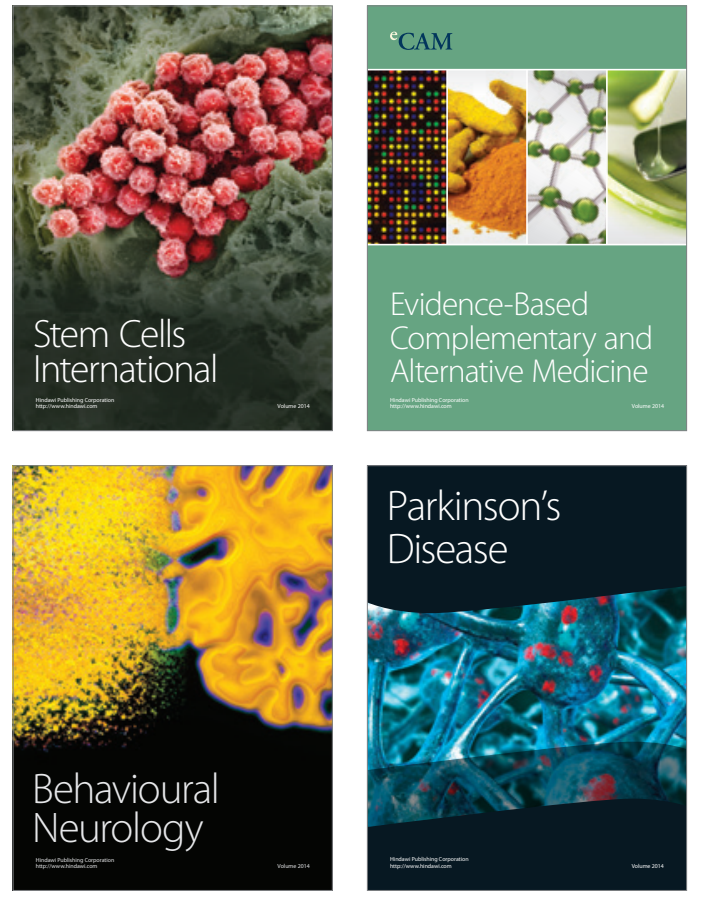
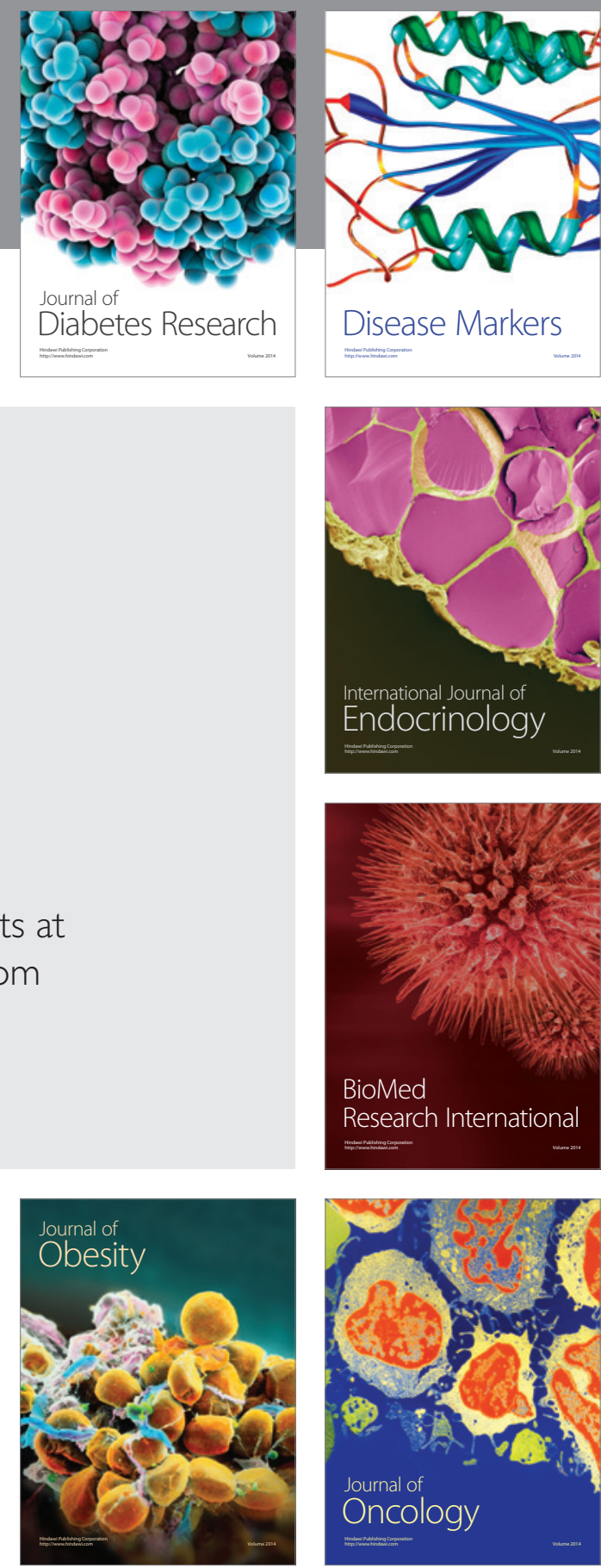

Disease Markers
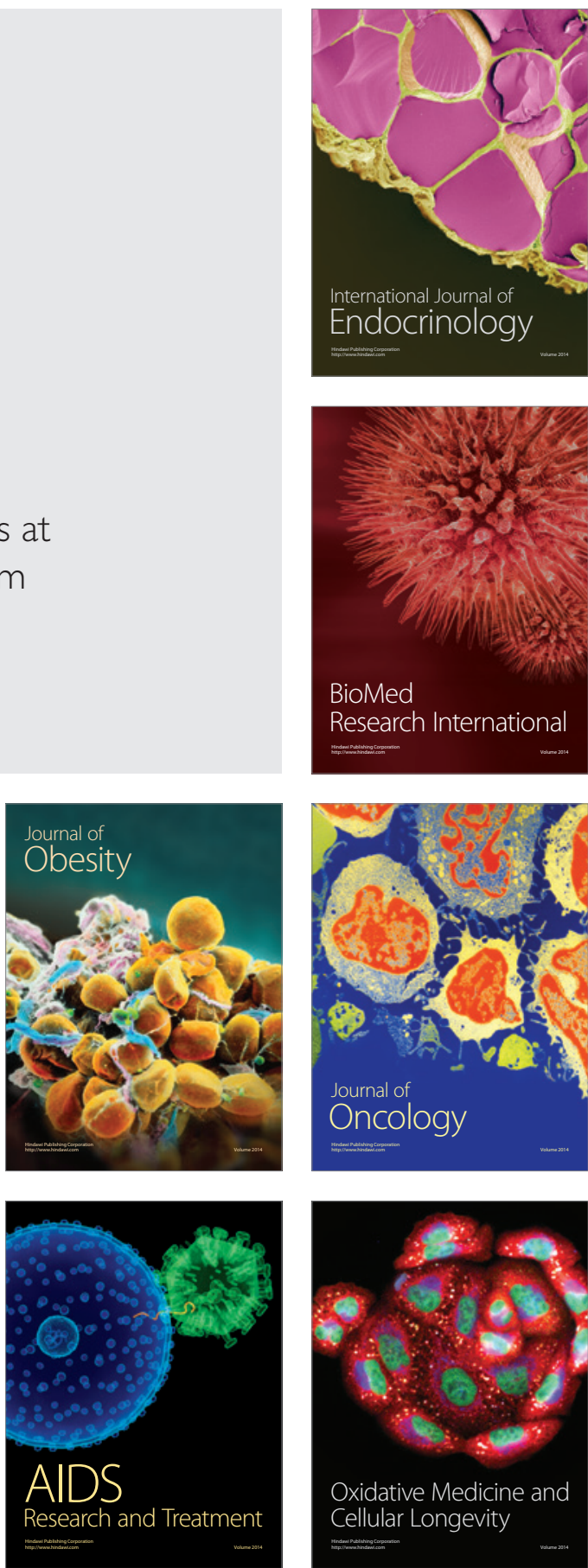